\title{
Granuloma eosinófilo de columna cervical
}

\author{
Felipe Gaudara S., 1 Ximena Gallegos A., Patricio O'Ryan Costa ${ }^{3}$ y Roberto Vigueras A. ${ }^{1}$
}

Eosinophitic Granuloma of the cervical spine

Two pátients with Eosinophilic Granuloma of the cervical spine are reported. The authors emphasize the rare localization of the tumor and a review od the literature in relation to histiocitosis $\mathrm{X}$ is made.

El granuloma eosinófilo es una enfermedad que se caracteriza por focos únicos o múltiples de osteolisis. Forma parte de un grupo de enfermedades denominadas histiocitosis $\mathbf{X}$, cuyo común denominador es el desarrollo de lesiones granulomatosas en hueso y tejidos blandos. Histopatológicamente se encuentra proliferación de histiocitos, eosinófilos y, ocasionalmente, células gigantes. Estas enfermedades serían diferentes expresiones de un mismo proceso patológico cuyo origen aún es desconocido.

La primera asociación entre estas enfermedades fue postulada por Lichtenstein en 1953, quien las clasificó en:

Fonnas localizadas: Granuloma eosinófilo.

Formas diseminadas: Aguda o subaguda: Enfermedad de Letterer-Siwe, con importante compromiso visceral, lesiones cutaneomucosas, infiltración medular. Forma maligna, de evolución fatal.

Crónica: Síndrome de Hand Schüller Christian, con manifestaciones óseas $y$ viscerales. Con cierta frecuencia existe invasión de hipotálamo e hipófisis con diabetes insipida y exoftalmo.

Evolución incierta, se ha descrito su resolución espontánea. ${ }^{\prime}$

El granuloma eosinóbilo representa aproximadamente el 50-60\% de este grupo de enfermedades. Tiene su máxima incidencia en las tres pri-

\footnotetext{
Becado en Pediatría.

2Médico en especialidad básica (Pediatría).

3 Médico Pediatra, Jefe de Sección Medicina Niños.

4 Neurocinujano.

Hospital Guillermo Grant Benavente, Concepcion.
}

meras décadas de la vida ${ }^{1,3,}$, A, 9 y hay franco predominio de sexo masculino $y$ de la raza blanca que ha becho sospechar una base genética." En general, los huesos del cráneo y extremidades son más frecuentemente afectados y los síntomas habituales son dolor, aumento de volumen e impotencia funcional en la zona de lesión. Se evoca con frecuencia un traumatismo previo a la aparición de las molestias y puede ser lo que permita descubrir el granuloma.

Se ha descrito la resolución espontánea de las lesiones únicas en un período variable, mientras se produce la reparación ósea. Por ello, algunos autores recomiendan una conducta expectante. Sin embargo, considerando la posibilidad de aparición multifocal, progresión a otras etapas o una ubicación de riesgo, se ha sugerido el uso de citostáticos, radioterapia, manejo quirúrgico del foco, e incluso inyección directa de corticoides en el sitio de lesión. ${ }^{8}$

Las siguientes circunstancias obligan a la intervención terapéutica del granuloma con el fin de acelerar la curación y evitar complicaciones.

- Lesión osteolítica en columna vertebral, con riesgo de compromiso de raíces nerviosas. Se han descrito paraplejías y otros grados de compromiso neurológico. ${ }^{2}$

- Dolor perseverante.

- Significativa restricción del movimiento o de la actividad física.

- Lesión radiológica muy agresiva o amenaza de compromiso del cartílago de crecimiento.

- Posibilidad de una fractura patológica. ${ }^{8}$

Se describen a continuación dos casos de granuloma eosinófilo de columna cervical que se pre- 
sentaron en nuestro hospital en 1980 , cuyo interés reside en la ubjcación poco frecuente de la lesión.

\section{Caso N. ${ }^{0}$ 1. A.R.S.}

Obs. $811.486,5$ años, peso $17,7 \mathrm{~kg}$, talla $1.05 \mathrm{~m}$. Sexo femenino.

Sin antecedentes patológicos de importancia.

Consulta después de 15 dias de dolor permanente en columna cervical irradiado al hombro derecho, anorexia, decaimiento. En el examen se encontró quejumbrosa, cuello en posición antíágica, contractura marcada de la musculatura cervical y dolor a la hiperextensión y flexión del cuello. No habia signos meníngeos, compromiso motorni sensitivo de las extremidades y el fondo del ojo era normal.

Hemograma, VHS, factor antinuclear, factor reumatoideo y $\mathbf{L C R}$ eran normales al ingreso. La radiografia de columna cervical demostró estenosis acentuada de los espacios C2-C3, C3-C4 y osteolisis del cuerpo de C3 (Fig. 1).

Fue tratada con reposo absoluto y tracción cervical.

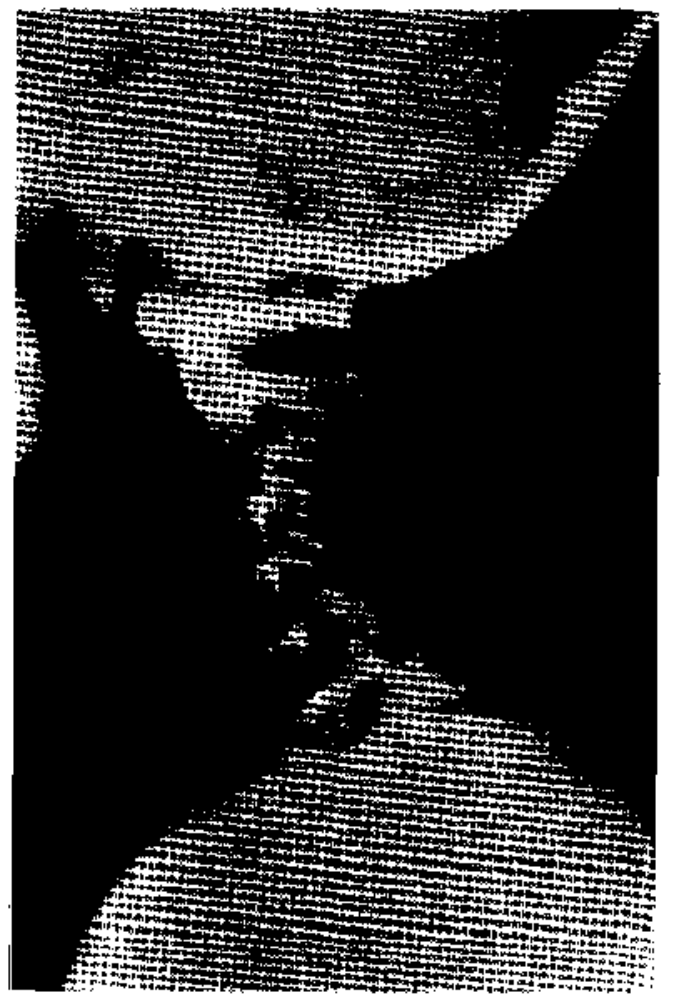

Figura 1
A] $6 .^{\circ}$ día de hospitalización se realizó biopsia corporal de C3. Posteriormente permaneció con collarete cervical y kinesiterapia, regresando parcialmente las manifestaciones clínicas.

La biopsia reveló GRANULOMA EOSINOFILO. Ante esto se tomaron radiografias de cráneo, resto de columna vertebral, pelvis y huesos largos, que fueron normales.

A los 44 días de su ingreso se practicó la resección quirúrgica de tejidos blandos entre $\mathrm{C} 2$ y C3, corporectomía parcial de $\mathrm{C} 3 \mathrm{y}$ artrodesis intercorporal C2-C3 con acrílico.

Una semana despuès, la radiografía cervical mostró expulsión del injerto de acrílico (Fig. 2). No habia compromiso neurológico. Se reintervino, efectuándose artrodesis intercorporal C2-C3 con autoinjerto peroneo fijado con acrílico. La biopsia tomada en esa intervención infomó: "Cartilago hialino con focos de degeneración, sin residuos de granuloma eosinófilo".

A Jos 62 dias de hospitalización, el control radiológico mostró nuevamente expulsión del injerto.

Se operó definitivamente dos meses y medio después del ingreso, practicándose corporecto-

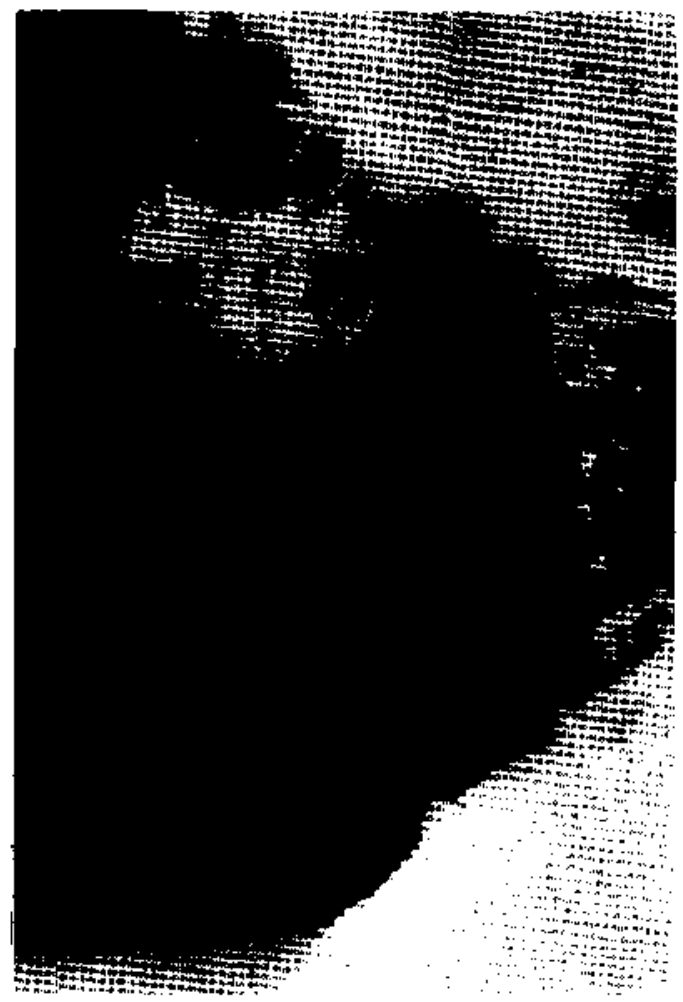


mía total de C3, y reemplazo del cuerpo vertebral con homoinjerto de ala iliaca. En la radiogratía 8 dias después se observó buena ubicación del injerto. En la biopsia reaparecieron células de granuloma eosinofilo, por lo que se inició radioterapia con una dosis de 2.000 rads sobre $\mathrm{C} 2-\mathrm{C} 3$ por un período de un mes.

Permaneció 132 días hospitalizada. Los controles radiológicos al alta $y$ hasta nueve meses después mostraron las artrodesis en buena posición. El examen neurológico es normal (Fig. 3).

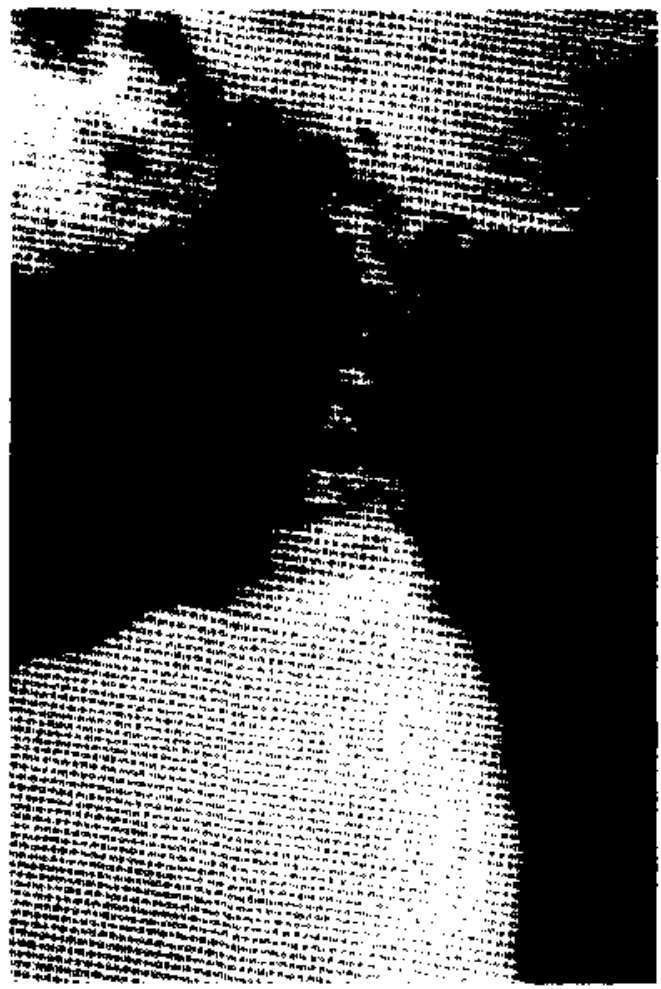

Figara 3

Caso N. ${ }^{\circ}$ 2, A.C.R.A.

Obs. $613.678,12$ años, $35 \mathrm{~kg}$, talla $1,40 \mathrm{~m}$. Sexo masculino. Sin antecedentes morbidos de importancia.

Su enfermedad se inició en los primeros días de marzo de 1980, a consecuencia de una torsión del cuello en clases de Educación Física, con dolor en la región cervicolateral derecha, progresivo, que limitaba los movimientos del cuello. Al repetirse el traumatismo en la zona se acentuaron las molestias, por lo que consultó en el hospital 15 días después. En la radiografía de columna cervical se comprob 6 fractura de $\mathrm{C} 4$ y destrucción del cuerpo vertebral (Fig. 4).

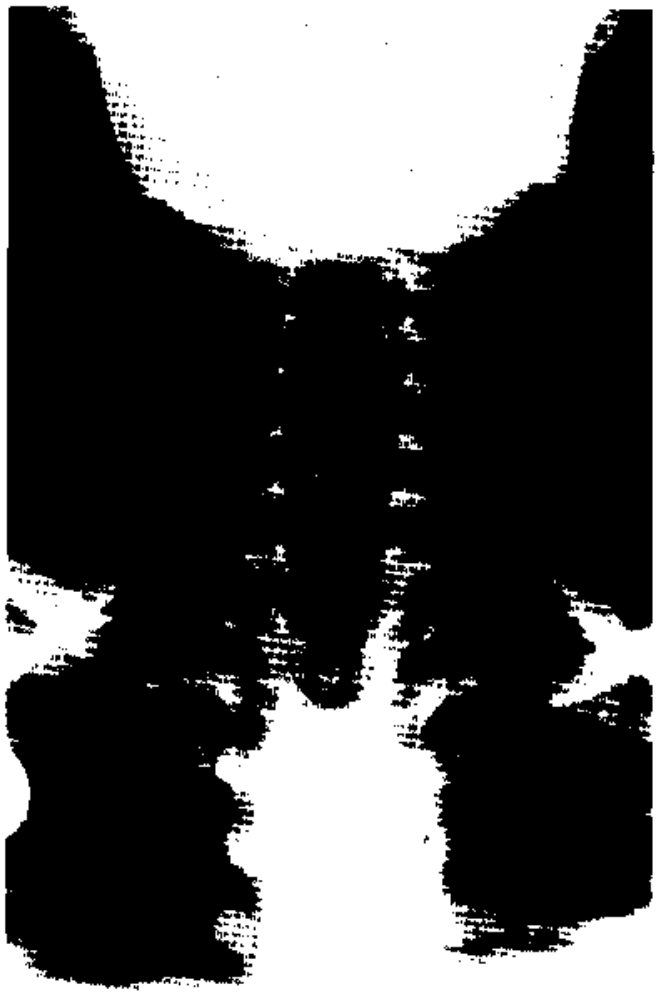

Figura 4

A pesar del reposo absoluto y collarete indicados, continuó con intenso dolor y bajo la sospecha de fractura patológica; fue intervenido dos semanas después. Se practic 6 corporectomja de C4 y se reemplazó el cuerpo de la vértebra con injerto de acrílico por vía anterior (Fig. 5). La evolución postoperatoria fue satisfactoria. El estudio anatomopatológico demostró un GRANULOMA EOSINOFILO. Las radiografías de huesos largos, cráneo, tórax, pelvis y columna fueron nomales.

Dos meses después de su ingreso inició tratamiento radioterápico con un total de 3.000 rads sobre la zona entre $\mathrm{C} 2$ y $\mathrm{C} 7$, que se prolongó por 24 días.

Se fue de alta en buenas condiciones generales con collarete hasta fines de 1980 . Los controles radiológicos posteriores demuestran que la artrodesis está en buenas condiciones (Fig. 6).

\section{COMENTARIO}

Se presentan dos casos de granuloma eosinófilo cervical, localización de escasa frecuencia. McCullough en 43 pacientes, sólo describe dos de ubicación en columna cervical, y es igualmente escasa en el resto de las publicaciones. 


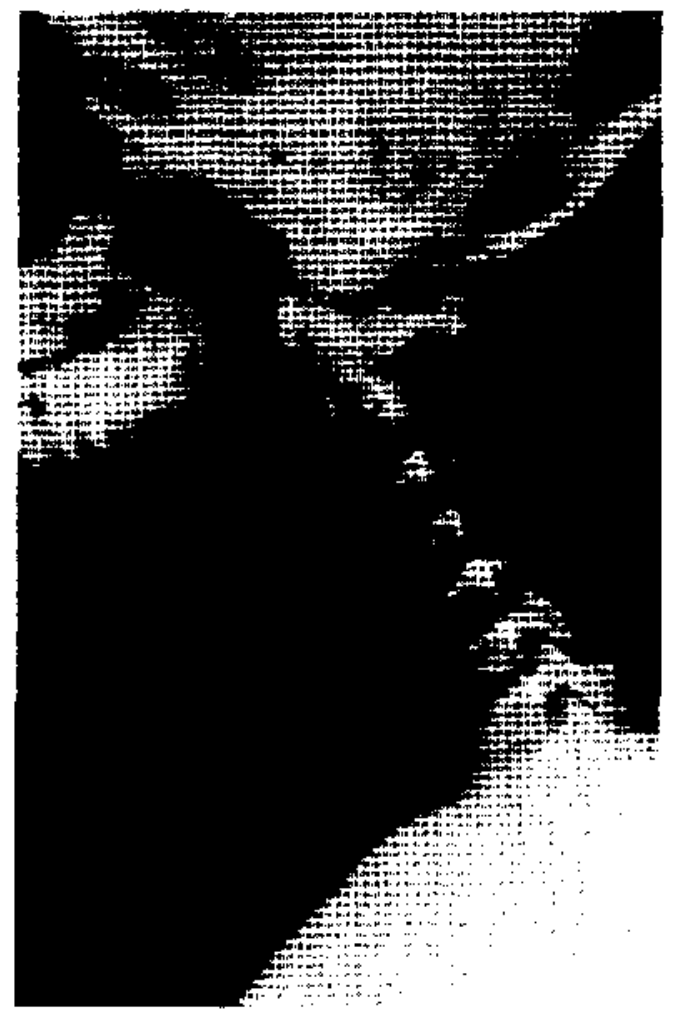

Figura 5

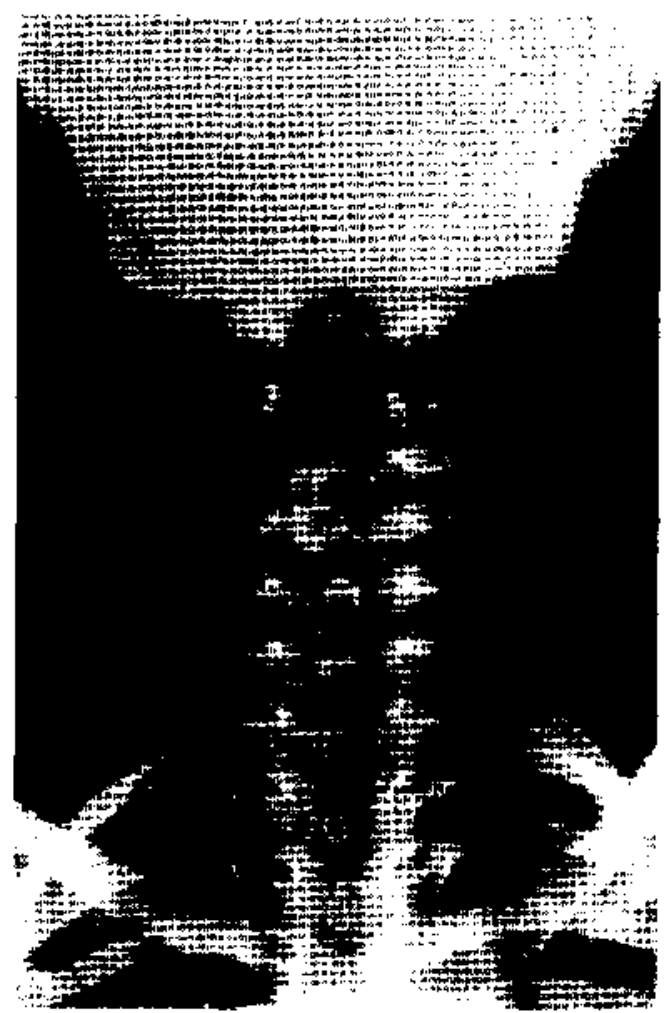

Es de interés de los autores llamar la atención sobre esta enfermedad poco frecuente en relación a los dolores cervicales, sean de aparición espontánea o de origen aparentemente traumático, $\mathrm{si}^{-}$ tuaciones ambas descritas en los casos clínicos presentados. Ante una lesión esquelética osteolitica debe sospecharse el diagnóstico. Una vez confinmado por el examen histopatológico, habrá que definir la extensión de la lesión para decidir, considerando los puntos mencionados anteriorinente, un tratamiento agresivo, quirúrgico si procede y radioterápico en Jos casos localizados. En los pacientes con lesiones multifocales se agrega la quimioterapia. ${ }^{5}$

Posteriormente deberá efectuarse un seguimiento estricto del paciente por un largo tiempo. ${ }^{5}$

El pronóstico está directamente relacionado con la localización y extensión de la enfermedad. En las formas localizadas, la mortalidad es de un $4 \%{ }^{5}$

\section{RESUMEN}

Se presentan dos pacientes con granuloma eosinófilo cervical. Se hace énfasis sobre la escasa frecuencia de esta localización y se revisa la literatura en relación al grupo de enfermedad llamadas histiocitosis $\mathrm{X}$.

\section{REFERENCIAS}

1 MacCulough, C. I. Eosinophilic Granuloma of bone. Acta Ortop. Scan, 51, 389-344, 1960.

2 Nell Green et al. Eosinophilic Granuloma of spine with as. sociated neural deficit. J. of Bone and Joint. Vol. 62-A, N." 7 , 1980.

"3 James Cinberg. Eosinophilic Granulorisa of head and neck. Five year review, Laryngomone. 88 : 1978.

4 Case records of Massachusetts Ceneral Hospital. Case 7 , I980. Eosin. Gran. of temporal Bune and ear caibal. The New England Joumal of Medicine, Feth. 21, 19tw.

5 Whim Zinkthom. Multifocal Ensinophilic Granulinum. The American Jourmal of Medicine. Vol. 6(1)4, aloril 1976.

6. Netson. IIistiocitosis X. Tratado de Pediatría. Tomo II

7 Rud Levy et ad. Eusinophilj: Gamulomia of temporal bone. Arch. Otolaryng. Yol. 106, miarzo 1980.

8 Mkchoel Cohen al. Direut injection of methylprednisolone sodium succitate in the treatment of solitary Eosinophilic Granuloma of bone. Report of 9 cases. Radiology 136: 289-243, ayosto 1950 .

9 Robert Sureet at. Eosinopluilic Graunlona in the temporal bone. Latryngoscupe. Vol. 89,0 ct. 1979. 